\title{
UNGEKLÄRTE FRAGEN ZU DEN HANDSCHRIFTEN CODEX CALIXTINUS UND RIPOLL 99
}

\author{
Verena FÖRSTER BINZ
}

\begin{abstract}
There are still a number of unanswered questions in connection with the Codex Calixtinus (E-SCc s.s.) They concern the origin of the compilation as it stands in the Codex Calixtinus and also partly in the manuscript Ripoll 99 (E-Bac 99), as well as the relationship between these two sources.

This article takes up observations which are based on a detailed comparison of the versions of the tunes from the Mass for the Feast of St. James which appears in both Mss. and of these two with manuscripts in the tradition of Aquitaine and Central France.

Several factors suggest that the original compilation was of Spanish or Southern French provenance and was edited and copied in Central France, also that the Mss. Ripoll 99 is neither a copy of the Codex Calixtinus nor based on a common original.
\end{abstract}

\section{Zusammenfassung}

Im Zusammenhang mit dem Codex Calixtinus (E-SCc s.s.) gibt es nach wie vor eine Reihe ungeklärter Fragen. Sie betreffen den Ursprung der im Codex Calixtinus und zum Teil auch in der Handschrift Ripoll 99 (E-Bac 99) überlieferten Kompilation sowie das Verhältnis zwischen diesen beiden Quellen.

Der vorliegende Beitrag nimmt Beobachtungen auf, die auf einem detaillierten Vergleich der Melodiefassungen der in beiden Handschriften enthaltenen Messe für das Hauptfest des Jacobus und auf einer Gegenüberstellung derselben mit Handschriften aus dem zentralfranzösischen und dem aquitanischen Überlieferungskontext basieren.

Mehrere Faktoren sprechen dafür, daß die ursprüngliche Kompilation spanischer oder südfranzösischer Provenienz war und in Zentralfrankreich redigiert und kopiert wurde und daß die Handschrift Ripoll 99 keine Abschrift des Codex Calixtinus ist und auch nicht auf der selben Vorlage basiert wie dieser.

Die Beschäftigung mit dem Codex Calixtinus ${ }^{1}$ führt fast unweigerlich zu einem Fragenkomplex, der in der Fachliteratur nach wie vor kontrovers diskutiert wird: Handelt es sich beim Codex Calixtinus um die Abschrift einer älteren, heute nicht mehr erhaltenen Handschrift ${ }^{2}$ oder ist er selbst als die erste vollständige Fassung des Inhaltes anzusehen? Ist die ganze Kompilation in den französischen oder in den spanischen Überlieferungskontext einzuordnen? Ist die Handschrift Ripoll $99^{3}$ eine Kopie des Codex Calixtinus oder nicht?

1. Santiago de Compostela, Archivo del Cabido de la Catedral, Codex Calixtinus, sin número (E-SCc s.s.). bezeichnet.

2. Die Urfassung dieser Kompilation wird seit José Bédier: Les légendes, Bd. III., S. 73, Anm. 1 als „Liber Sancti Iacobi“

3. Barcelona, Arxiu de la Corona d'Aragon, Ripoll 99 (E-Bac 99). 
Den Ausgangspunkt für die bisherige Auseinandersetzung mit dieser Thematik bilden zwei gegensätzliche Ansichten zum Ursprung des Codex Calixtinus, die bis in die jüngste Zeit von verschiedenen Wissenschaftlern vertreten werden:

Die eine These stammt von Peter Wagner, der innerhalb des Codex Calixtinus mehrere Ungereimtheiten in inhaltlicher, liturgischer und musikalischer Hinsicht feststellte. Er schloß daraus, daß „der Codex von Santiago [...] nicht das Original der Jakobusliturgie sein“ könne, sondern die Kopie einer anderen, nicht mehr erhaltenen Handschrift des „Liber Sancti Iacobi“ sein müsse, von dem es bereits im 12. Jahrhundert mehrere Abschriften gab ${ }^{4}$. Klaus Herbers und Christopher Hohler berücksichtigten zwar die Aussagen Wagners nicht, kamen aber aufgrund eigener Untersuchungen zum gleichen Ergebnis ${ }^{5}$.

Die andere These ist rund zwanzig Jahre jünger und stammt von Adalbert Hämel, der Wagners Überlegungen zwar zunächst aufgriff, ihnen aber keine Beweiskraft zugestand. Von vorne herein ging er davon aus, daß der Codex Calixtinus mit dem „Liber Sancti Iacobi“ identisch sei. ${ }^{6}$ Aufgrund eigener Untersuchungen auf inhaltlicher und sprachlicher Ebene gelangte er allerdings zu der Ansicht, daß diese Urfassung des Inhaltes ,auf älterem Material [beruhe], das nur vom Kompilator erweitert und für seine Zwecke umgestaltet“" worden sei $^{7}$. Die Ungereimtheiten innerhalb der Kompilation begründete er angesichts paläographischer Befunde damit, daß der Codex nachträglich stark verändert worden sei und heute nicht mehr in seiner ursprünglichen Form vorliege ${ }^{8}$. André Moisans Ansichten korrespondieren mit den Aussagen Hämels ${ }^{9}$, wofür er von Michel Huglo in seiner Rezension scharf kritisiert wurde ${ }^{10}$.

Für den „Liber Sancti Iacobi“ bzw. den Inhalt der Kompilation wurde von den meisten Forschern ein französischer Ursprung angenommen. Diese Vermutung stützt sich auf inhaltliche Aspekte, wie beispielsweise auf die mehrfache Erwähnung und zum Teil genaue Beschreibung einiger französischer Orte wie etwa Cluny und Saint-Denis, oder auf den Umstand, daß im Pilgerführer keine spanische Route, sondern nur Wegstrecken, die aus Frankreich nach Santiago de Compostela führen, angegeben sind ${ }^{11}$. Die wenigsten Autoren zogen überhaupt spanische Einflüsse in Erwägung ${ }^{12}$. Allein Hohler vermutete, daß die Urfassung in Spanien - wohl in direktem Zusammenhang mit Santiago de Compostela - entstand. Die Kompilation sei später als Schulbuch im Umkreis von Saint-Denis, möglicherweise im Cluniazenser Kloster Saint-Martin-de-Champs, verwendet $^{13}$ und $\mathrm{zu}$ diesem Zweck von einem Geistlichen, der sich mit Spitznamen „Calixtus“

4. Wagner: Die Gesänge, S. 6, 9, 132. Eine größere Anzahl solcher Quellen hat sich bis in unsere Zeit erhalten. Doch, soweit bekannt, sind nur im Codex Calixtinus und in der Handschrift Ripoll 99 notierte Gesänge enthalten. Zu den weiteren Überlieferungen des „Liber Sancti Iacobi“" grundlegend Hämel: Überlieferung, S. 21-44.

5. Herbers: Der Jakobuskult, S. 22 diskutiert u.a. die Thesen von José Bédier, Pierre David und René Louis und räumt auch Hämels Überlegungen einen großen Stellenwert ein; Hohler: „A Note“, S. 39, 62/63.

6. Hämel: Überlieferung, S. 5.

7. Hämel: Überlieferung, S. 51 .

8. Ebd., S. 11.

9. Moisan: Le livre, S. 43. Er bezieht sich hier auf Bédier: Les légendes, Bd. III, S. 367, 448.

10. Moisan: Le livre, S. 39-40, 43 und Huglo: Rezension, S. 260.

11. Moisan: Le livre, S. 59/60, 63; Hämel: Überlieferung, S. 57, 59, 60.

12. Diaz y Diaz: El codice, S. 90/91; López-Calo: La musica, S. 91.

13. Hohler: „A Note“, S. 39. 
genannt habe, bearbeitet worden ${ }^{14}$. Ein sich nach dem Kanzler des Papstes Calixtus II. „Aimericus“ nennender Lehrer habe das Buch schließlich nach Vézelay gebracht und dort für den Unterricht verwendet $^{15}$. In der Folge seien mehrere Kopien angefertigt worden, wodurch das Werk eine weitere Verbreitung gefunden habe und schließlich wieder zurück nach Spanien gelangt sei. Die Tradition dieser überarbeiteten Texte sei jedoch rein französisch ${ }^{16}$. Diese auf den ersten Blick sehr kühn anmutenden Überlegungen fanden in den nachfolgenden Publikationen nur geringe Beachtung. So attestierte beispielsweise Herbers Hohler, daß er ,viele bisher kontrovers behandelte Fragen einer völlig neuartigen Lösung“ zuführe, tat seinen Text aber insgesamt lediglich als „,anregende Lektüre“ $\mathrm{ab}^{17}$.

Auch zum Verhältnis zwischen Codex Calixtinus und Ripoll 99 liegen zwei gegensätzliche Thesen vor, die beide auf Unterschiede in der Überlieferung des Inhaltes zurückgeführt werden:

Wagner stellte angesichts zahlreicher Abweichungen zwischen den Melodiefassungen der in beiden Handschriften vollständig notierten Hauptmesse zum Jacobusfest - die Vigilmesse ist in Ripoll 99 nicht enthalten - die Frage, ob dem Kopisten tatsächlich der heutige Codex vorlag oder ob er eine andere Quelle benutzte, relativierte diese Vermutung aber gleich wieder, indem er auch in Erwägung zog, daß Arnaldus, der Schreiber von Ripoll 99, die Melodien ,in der Fassung niederschrieb, die in seinem Kloster für die älteren Messen üblich war. " ${ }^{18}$ Auch Hohler und Huglo vertraten die Ansicht, daß Ripoll 99 die Kopie einer anderen Handschrift des „Liber Sancti Iacobi“ sein müsse ${ }^{19}$.

Hämel verglich den Inhalt beider Handschriften - nicht aber die Melodiefassungen - und vermutete, daß Ripoll 99 eine Kopie des Codex Calixtinus sei. Er führte die Unterschiede zwischen beiden Quellen auf den Umstand zurück, daß der Schreiber von Ripoll 99 noch den ursprünglichen Codex vor sich gehabt habe ${ }^{20}$. Ebenso hielten Klaus Herbers, Elisardo Temperàn Villaverde, José López-Calo und Sarah Fuller Ripoll 99 für eine Kopie des Codex Calixtinus ${ }^{21}$.

Angesichts der zahlreichen Untersuchungen zu diesem Themenkreis scheint es zunächst müßig, die oben gestellten Fragen noch einmal aufzunehmen. Doch liegt den folgenden Beobachtungen der Versuch zugrunde, auf einem neuen Weg zu Antworten zu gelangen ${ }^{22}$ :

14. Ebd., S. 33.

15. Ebd., S. 56/57. In der „Epistola domni pape Innocentii“ im Anhang des Codex Calixtinus (fol. 192r) wird ein Aimeric(us) Picaud(us) als Überbringer des Codex bezeichnet, der sich auch unter den Unterzeichnern findet, die die Echtheit des Buches bestätigen. Zur Person des Aymericus sowie zu anderen Zuschreibungen innerhalb des Codex unter Berücksichtigung der älteren Literatur Lütolf: Mehrstimmige Ordinarium Missae-Sätze, Bd. I, S. 81-86; vgl. auch Herbers: Der Jakobuskult, S. 36; Moisan: Le Livre, S. 63.

16. Hohler: „A Note“, S. 37, 61/62.

17. Herbers: Der Jakobuskult, S. 46.

18. Wagner: Die Gesänge, S. 9. Generell zu Ripoll 99 Wagner: Die Gesänge, S. 14; Hämel: Überlieferung, S. 22; Moisan: Le livre, S. 73, 85; Huglo: „The Origin“, S. 198; López-Calo: La Musica, S. 83.

19. Hohler: „A Note“, S. 70. Huglo: „Les Pièces notées“, S. 110; ders.: „The Origin“, S. 78.

20. Hämel: Überlieferung, S. 22-28.

21. Herbers: Der Jakobuskult, S. 33; Villaverde: La liturgia, S. 35; López-Calo: La musica, S. 88; ders.: „Claves“, S. 235; Fuller: „Perspectives“, S. 209, 211.

22. Der vorliegende Beitrag beruht auf Teilaspekten aus meiner 2001 vorgelegten Basler Lizentiatsarbeit „Zwischen Alt und Neu - Die beiden notierten Messen des Codex Calixtinus in ihrem Kontext". 
Bereits Wagner erkannte, daß die meisten Gesänge der Jacobusliturgie unter Verwendung älterer Melodien formuliert wurden und wies die Herkunft des größten Teils der Vorlagen nach ${ }^{23}$. Doch wurde nach möglichen Kriterien für die Gestaltung der neuen Meßformulare bis jetzt nicht gefragt. Dieser Faktor ist aber insofern von Bedeutung, als die Auswahl der Melodievorlagen jeder Kompilation vorangegangen sein muß. Da davon auszugehen ist, daß die Kompilatoren durch die Tradition ihres Umfeldes geprägt waren, könnten die verwendeten Melodievorlagen einen Schlüssel zur Frage nach der Herkunft dieser Kompilation bieten.

Auch die melodischen Abweichungen zwischen beiden Überlieferungen der Hauptmesse sind bereits seit 1931 zum allergrößten Teil in Wagners Redaktion der Gesänge des Codex Calixtinus ${ }^{24}$ greifbar. Ein detaillierter Vergleich und eine Einordnung beider Melodiefassungen in einen Überlieferungskontext stehen noch aus. Doch gerade diese beiden Vorgehensweisen könnten zu weiteren Hinweisen bezüglich des Verhältnisses zwischen den beiden Quellen und ihrer Provenienz führen.

Beide Messen der Jacobusliturgie enthalten Gesänge, die mit ihrem alten Text und ihrer alten Melodie übernommen, Gesänge, die neu textiert sowie solche, die neu komponiert wurden ${ }^{25}$ :

$$
\begin{aligned}
& \mathrm{A}=\text { alte Melodie und alter Text } \\
& \mathrm{B}=\text { alte Melodie neu textiert } \\
& \mathrm{C}=\text { neu komponiert }
\end{aligned}
$$

\begin{tabular}{|c|c|c|c|}
\hline \multirow[t]{2}{*}{ Incipit } & \multicolumn{3}{|c|}{ Musik-Text } \\
\hline & $\mathbf{A}$ & B & $\mathbf{C}$ \\
\hline Intr. „Iacobus et Iohannes“ & & $\mathrm{x}$ & \\
\hline V. „Iam vos delectat“" & & $\mathrm{x}$ & \\
\hline Tract. „Vocavit Ihesus“ & & $\mathrm{x}$ & \\
\hline Grad. „Nimis honorati“ & $\mathrm{x}$ & & \\
\hline Tract. „Iacobus in vita“ & & $\mathrm{x}$ & \\
\hline Off. „Certe dum filii““ & & $\mathrm{x}$ & \\
\hline V. „Iam locum celsitudinis“ & & $\mathrm{x}$ & \\
\hline Com. „Ego vos elegi“ & $\mathrm{x}$ & & \\
\hline
\end{tabular}

Vigilmesse (Codex Calixtinus, fol. 114r-116v):

23. Wagner: Die Gesänge, S. 144-145 und ders.: Einführung, Bd. III, S. 421.

24. Wagner: Die Gesänge, S. 78-92.

25. Neben den hier behandelten Propriumsgesängen finden sich im Codex Calixtinus die beiden Prosen „Gratulemur et letemur“ und „Clemens servulorum“. Die Handschrift Ripoll 99 enthält nur die letztere. Bei „Gratulemur et letemur“" handelt es sich um die gekürzte Fassung eines Gesanges, der sich in einigen weiteren Quellen des 12. Jahrhunderts findet. „Clemens servulorum“ dürfte eine Neukomposition sein (vgl. Wagner: Die Gesänge, S. 146 Anm. 2, S. 147). Da „Gratulemur et letemur" in einer für eine Gegenüberstellung geeigneten Fassung bis jetzt nur in sehr wenigen Handschriften nachgewiesen werden konnte, wurde hier auf eine Untersuchung verzichtet. 
Hauptmesse (Codex Calixtinus, fol. 118r-122r; Ripoll 99 fol. 31v-33r):

\begin{tabular}{|c|c|c|c|}
\hline \multirow[t]{2}{*}{ Incipit } & \multicolumn{3}{|c|}{ Musik-Text } \\
\hline & $\mathbf{A}$ & & $\mathbf{C}$ \\
\hline $\begin{array}{l}\text { Intr. ,Ihesus vocavit““ } \\
\text { V., „Celi enarrant“" } \\
\end{array}$ & $\mathrm{x}$ & $\mathrm{x}$ & \\
\hline Grad. „Misit Herodes“ & & $\mathrm{x}$ & \\
\hline All. „Sanctissime apostole“ & & $\mathrm{x}$ & \\
\hline All. „Hic Iacobus“ & & $\mathrm{x}$ & \\
\hline All. „Vocavit Ihesus“" & & & $\mathrm{x}$ \\
\hline $\begin{array}{l}\text { Off. „Ascendens Ihesus“" } \\
\text { V. „Et enim sagitte“ }\end{array}$ & & $\mathrm{x}$ & $\mathrm{X}$ \\
\hline $\begin{array}{l}\text { Com. „Ait Ihesus“ } \\
\text { V. „Si mens vestra appetit“ }\end{array}$ & & $\begin{array}{l}\mathrm{x} \\
\mathrm{x}\end{array}$ & \\
\hline
\end{tabular}

Nur für das dritte Alleluia „Vocavit Ihesus“ und den Offertoriumsvers „Et enim sagitte“ aus der Hauptmesse wurde die Melodie neu komponiert ${ }^{26}$. Das Graduale „Nimis honorati“ und die Communio „Ego vos elegi“ aus der Vigilmesse wurden mit ihrem ursprünglichen Text und ihrer ursprünglichen Melodie aus anderen Meßformularen übernommen. Der ebenfalls einen älteren Text aufweisende Introitusvers „Celi enarrant“ aus der Hauptmesse wird entsprechend dem Modus der Antiphon nach einem Modell der Psalmodie im siebten Ton gesungen und ist nur als Incipit notiert. Bei allen übrigen Gesängen handelt es sich um Neutextierungen bzw. Adaptionen. Über die melodischen Grundlagen der Gesänge, die auf ältere Melodien zurückgehen, vermittelt die folgende Tabelle einen Überblick:

\section{Vigilmesse:}

\begin{tabular}{|c|c|}
\hline Incipit & Vorlage \\
\hline $\begin{array}{l}\text { Intr. „Iacobus et Iohannes“" } \\
\text { V. „Iam vos delectat“" }\end{array}$ & $\begin{array}{l}\text { Intr. „Lux fulgebit“" } \\
\text { V. „Dominus regnavit““ }\end{array}$ \\
\hline Tract. „Vocavit Ihesus“ & Tract. „Cantemus domino“ / „Sicut cervus“ \\
\hline Grad. „Nimis honorati““ & Grad. „Nimis honorati““ \\
\hline Tract. „Iacobus in vita sua“ & Tract. „Vinea facta“/ „Adtende celum“ \\
\hline $\begin{array}{l}\text { Off. „Certe dum filii““ } \\
\text { V. „Iam locum celsitudinis““ }\end{array}$ & $\begin{array}{l}\text { Off. „Posuisti domine” / „Angelus domini” } \\
\text { V. „Magna est gloria“/,,Ihesus stetit“" }\end{array}$ \\
\hline Com. „Ego vos elegi“ & Com. „Ego vos elegi““ \\
\hline
\end{tabular}

26. Wagner (Die Gesänge, S. 145) geht irrtümlich davon aus, daß das zweite und dritte Alleluia neu komponiert wurden, den Offertoriumsvers zieht er nicht als Neukomposition in Betracht. Doch lassen sich beide Gesänge aufgrund formaler und melodischer Charakteristika als Neukompositionen identifizieren. 


\section{Hauptmesse:}

\begin{tabular}{|c|c|}
\hline Incipit & \begin{tabular}{|l|} 
Vorlage \\
\end{tabular} \\
\hline $\begin{array}{l}\text { Intr. „Ihesus vocavit" } \\
\text { V. „Celi enarrant"“ }\end{array}$ & $\begin{array}{l}\text { Intr. „In virtute“ / „Probasti domine“ } \\
\text { V. „Celi enarrant““ }\end{array}$ \\
\hline Grad. „Misit Herodes“ & $\begin{array}{l}\text { Grad. „Ecce sacerdos” / „Exiit sermo” / } \\
\text { „Christus factus est“/ (,,Misit dominus“) }\end{array}$ \\
\hline All. „Sanctissime apostole“ & $\begin{array}{l}\text { All. „Letabitur iustus” / „Levita Laurencius” / } \\
\text { („Diffusa est“) / („Concussum est“) }\end{array}$ \\
\hline All. „Hic Iacobus“ & All. „Iste sanctus digne” \\
\hline Off. „Ascendens Ihesus“ & $\begin{array}{l}\text { Off. „Iustorum anime“"/,„Stetit angelus““/ (,Tu } \\
\text { es Petrus“) / (,,Viri galilei“) }\end{array}$ \\
\hline $\begin{array}{l}\text { Com. „Ait Ihesus“ } \\
\text { V. „Si mens vestra appetit“" }\end{array}$ & $\begin{array}{l}\text { Com. „Tu es Petrus““ } \\
\text { V. „Et porte inferi““ }\end{array}$ \\
\hline
\end{tabular}

Wagners Zuordnung der Gesänge und ihrer Vorlagen wurde aufgrund von Hinweisen bei Karlheinz Schlager ${ }^{27}$ und F. Javier Lara ${ }^{28}$ sowie durch eigene Befunde ergänzt. In mehreren Fällen nennt die obige Tabelle nicht nur einen, sondern mehrere Gesänge, die jeweils auf das gleiche melodische Material zurückzuführen sind und deshalb als Basis für die Gesänge des „Liber Sancti Iacobi“" in Frage kommen. Die Klammern weisen auf Gesänge hin, die ebenfalls auf die gleiche Melodie zurückgehen, aber in wesentlichen Punkten von den für die Jacobusliturgie verwendeten Fassungen abweichen.

Zur Frage nach der Entstehung neuer Meßformulare im späten Mittelalter legten bereits in den 20er bis 40er Jahren des 20. Jahrhunderts Peter Wagner und Domenicus Johner grundlegende Beobachtungen vor ${ }^{29}$. Johner untersuchte primär die Situation im 10. Jahrhundert anhand des Codex 339 aus St. Gallen, der neben dem alten, sanktionierten Repertoire auch neuere Feste enthält, die aber hauptsächlich auf älteres Material zurückgreifen und nur wenige neue Gesänge aufweisen. Er übertrug seine Beobachtungen auf die Situation des 12. Jahrhunderts, also auf den zeitlichen Kontext, in dem die Handschriften Codex Calixtinus und Ripoll 99 entstanden sind. Dabei wies er darauf hin, daß jeweils „eine noch größere Ehrfurcht vor den überlieferten Melodien“ gewahrt wurde als in früherer Zeit ${ }^{30}$. Zum gleichen Schluß kam auch Wagner im Zusammenhang mit der Meßliturgie für den Hl. Martinus von Tours ${ }^{31}$.

27. Schlager: Alleluia-Melodien, Bd. II, S. 833.

28. Lara: „Estructuras“, S. 284-287. Außer den Offertorien „Posuisti domine“ und „Angelus domini“ gaben Wagner und Lara „Ave Maria“ an. In diesem findet sich in der Antiphon bei „Dominus tecum“ ein Abschnitt, der mit einem Melisma im Vers von „Certe dum filii“" (über „celsitudinem“) verwandt ist und auch im - je nach Handschrift - ersten oder zweiten Vers von „Angelus domini“ und „Posuisti domine“ erscheint. Wagner (Einführung, Bd. III, S. 425) wies nach, daß dieses Melisma erst später den zuerst fehlenden Worten „Dominus tecum“ unterlegt wurde und nicht zum ursprünglichen Gesang gehört. Da sich keine weiteren Beziehungen zwischen „Certe dum filii“" und „Ave Maria“ feststellen lassen, wird dieser Gesang hier nicht als Vorlage in Betracht gezogen.

29. Wagner: „Wie man im Mittelalter“; Johner: Wort und Ton, S. 454-464.

30. Johner: Wort und Ton, S. 463.

31. Wagner: „Wie man im Mittelalter“, S. 147/148, 168/169. 
Analog zu Johners Überlegungen ist denkbar, daß Bezüge zwischen alten Gesängen und dem Jacobusfest bzw. seinem unmittelbaren zeitlichen Umkreis als Auswahlkriterium für die Melodien gedient haben könnten ${ }^{32}$.

Für die diesen Beobachtungen zugrundeliegende Untersuchung wurden achtzehn Quellen herangezogen, die im späten 11. bis ins ausgehende 12. Jahrhundert in Frankreich und Spanien entstanden sind. Die Auswahl berücksichtigt die unterschiedliche Notation der beiden Hauptquellen und stellt Beziehungen zum Umkreis von Orten her, die im Zusammenhang mit der Jacobusliturgie eine Rolle spielen: Die auf vier Linien notierten Neumen des Codex Calixtinus weisen nach Zentralfrankreich, Ripoll 99 enthält aquitanische Neumen, die nicht auf gezogenen, sondern auf geritzten Linien notiert $\operatorname{sind}^{33}$. Die Neumen des Codex Calixtinus ähneln denen der Handschriften F-Pn nouv. acq. lat. 1235 (Graduale aus Nevers) und F-Pn lat. 10511 (Graduale aus Auxerre), wobei die Herkunftsorte beider Quellen nicht mehr als $50 \mathrm{~km}$ von Vézelay entfernt sind, wo der mutmaßliche Überbringer des Codex, Aimery Picaud, gelebt haben soll. Etwa $100 \mathrm{~km}$ beträgt die Strecke von Troyes nach Vézelay. Maillezais liegt im Poitou, der Region, aus der Aimery Picaud stammte, und ist etwa $50 \mathrm{~km}$ von dessen Heimatort Parthenay entfernt. Die Entfernung zwischen Angers und Parthenay beträgt ungefähr $100 \mathrm{~km}$. Villeneuve-lez-Avignon ist etwa $40 \mathrm{~km}$ und Narbonne ungefähr $100 \mathrm{~km}$ von Saint-Gilles entfernt, das im Codex mehrmals erwähnt wird. Auch Saint-Denis wird häufig genannt. Nachdem aufgrund von Hinweisen in den Texten bereits mehrfach vermutet wurde, daß der Autor des „Liber Sancti Iacobi“ ursprünglich aus Cluny stammen könnte ${ }^{34}$, wurden auch einige Handschriften herangezogen, die mit den Cluniazensern in Verbindung stehen:

\title{
Zentralfranzösische Quellen:
}

\author{
F-AN 97 (89), Angers \\ F-Pm 384, Saint-Denis \\ F-Pn lat. 9435, Saint-Pierre de Maillezais, Poitou \\ F-Pn lat. 9437, Troyes \\ F-Pn lat. 10511, Saint-Cyran-en Braine bei Auxerre \\ F-Pn lat. 12584, Saint-Maur-des-Fossés
}

32. Johner: Wort und Ton, S. 461.

33. Vgl. Wagner: Die Gesänge, S. 11, 14; Lütolf: Mehrstimmige Ordinarium Missae-Sätze, Bd. I, S. 87/88 Anm. 63, S.89; Huglo: „The Origin“, S. 196-198; López-Calo: La Musica, S. 78/79, 84; Moisan: Le Livre, S. 85; Fuller: „Perspectives“, insbesondere S. 203-213. Bei den Provenienz- und Datierungsangaben stütze ich mich auf Graduel Romain, Bd. II und Hiley: Art. „Sources“, S. 824-839, bezüglich der Quellen, die mit Cluny in Verbindung gebracht werden, auch auf Huglo/Ferreira: Art. „Cluniac monks“, S. 63/64. Für die spanischen Quellen beziehe ich mich auch auf Anglès: La música.

34. Lütolf: Mehrstimmige Ordinarium Missae-Sätze, Bd. I, S. 88; Herbers: Der Jakobuskult, S. 34, mit der Diskussion der älteren Literatur. Vgl. auch Moisan: Le Livre, S. 59, 63; Hohler: „A Note“, S. 47. Dieser Zusammenhang wird angenommen, obwohl es sich aufgrund der Zahl der Antiphonen und Responsorien des Offiziums um keine monastische, sondern um eine weltkirchliche Liturgie handelt. Ausschlaggebend ist lediglich die häufige Nennung von Cluny im Codex. 
F-Pn lat. 17328, Paris

F-Pn nouv. acq. lat. 1235, Nevers

F-Psg 93, Paris

\section{Aquitanische Quellen:}

B-Br II 3823, Sauxillanges, Auvergne (Cluniazenser)

E-Mah Aemil. 18, S. Millán de la Cogolla ${ }^{35}$

E-SA 2637, Salamanca (?)

F-A 181, Villeneuve-lez-Avignon

F-Pn lat. 776, Saint-Michel-de-Gaillac bei Albi

F-Pn lat. 780, Narbonne

F-Pn lat. 903, Saint-Yrieix bei Limoges

F-Pn lat. 1087, Cluny

GB-Lbl Harley 4951, Toulouse

Es zeigte sich, daß zum Jacobusfest (25. Juli) die beiden Gesänge, die mit altem Text und alter Melodie übernommen wurden - das Graduale „Nimis honorati“ und die Communio „Ego vos elegi“"-, sowie der Introitusvers „Celi enarrant“ an mehreren Orten erklangen:

\section{Zentralfranzösische Notation:}

\begin{tabular}{|l|l|}
\hline F-Pn lat. 10511 (fol. 196r) & Com. „Ego vos elegi““ \\
\hline F-Pn lat. 12584 (fol. 189r) & Grad. „Nimis honorati“ \\
\hline F-Pn lat. 17328 (fol. 137r) & Grad. „Nimis honorati“ \\
\hline F-Psg 93 (fol. 163v) & Com. „Ego vos elegi““ \\
\hline
\end{tabular}

35. Bei Meßformularen, die in dieser Handschrift keine Gesänge enthalten, wurde auf die Quelle E-Mah Aemil. 51 zurückgegriffen, ein Graduale, das aus dem gleichen Kloster stammt und ebenfalls im 11./12. Jahrhundert entstand. 


\section{Aquitanische Notation:}

\begin{tabular}{|l|l|}
\hline E-Mah Aemil. 18 (fol. 266v) ${ }^{36}$ & $\begin{array}{l}\text { Intr.-v. ,Celi enarrant” } \\
\text { Grad. ,Nimis honorati” }\end{array}$ \\
\hline E-SA 2637 (fol. 203 r/v) & Grad. „Nimis honorati” \\
\hline F-A 181 (fol. 101v/102r) & $\begin{array}{l}\text { Grad. „Nimis honorati” } \\
\text { Com. „Ego vos elegi” }\end{array}$ \\
\hline F-Pn lat. 776 (fol. 105v) & $\begin{array}{l}\text { Intr.-v. ,Celi enarrant” } \\
\text { Grad. ,Nimis honorati” }\end{array}$ \\
\hline F-Pn lat. 780 (fol. 96v) & Grad. „Nimis honorati” \\
\hline F-Pn lat. 903 (fol. 105r/S. 209) & $\begin{array}{l}\text { Intr.-v. ,Celi enarrant } \\
\text { Grad. ,Nimis honorati” }\end{array}$ \\
\hline F-Pn lat. 1087 (fol. 78r) & Grad. ,Nimis honorati” \\
\hline GB-Lbl Harley 4951 (fol. 275v) & Grad. ,Nimis honorati” \\
\hline
\end{tabular}

Wie die obige Tabelle verdeutlicht, lassen sich wesentlich mehr Beziehungen zu den aquitanischen als zu den zentralfranzösischen Quellen feststellen. Auch geht aus der Zusammenstellung hervor, daß das Graduale „Nimis honorati“ offensichtlich im aquitanischen Raum zum festen Bestandteil des Jacobusfestes gehörte. In der einleitenden Epistel des Codex Calixtinus und der Handschrift Ripoll 99, die die Gesänge aufzählt, die vor der Einführung der neuen Liturgie in Santiago de Compostela zum Jacobusfest gesungen wurden, wird zwar „Nimis honorati“ nicht explizit genannt, da jeweils nur auf die Introitus der Messen hingewiesen wird, doch erscheint hier der Introitus „Michi autem nimis“38. Dieser wurde nach den verschiedensten französischen und spanischen Quellen an mehreren Apostelfesten (z.B. zu Thomae Apostoli, Bartholomei Apostoli, Simonis et Judae Apostoli, Andreae Apostoli) gesungen und erscheint fast immer in Kombination mit dem Graduale „Nimis honorati“39. So darf vermutet werden, daß die Kompilatoren mit den liturgischen Gepflogenheiten in Santiago de Compostela vertraut waren und bewußt einen Gesang beibehielten, der dort bereits mit dem Jacobusfest assoziiert wurde.

Auffällig ist auch, daß alle drei Handschriften, die am Jacobusfest den Introitusvers der Hauptmesse „Celi enarrant" enthalten, spanischer und südfranzösischer Herkunft sind ${ }^{40}$. Dieser Vers ist in keiner der herangezogenen Vergleichsquellen mit den Melodievorlagen von "Ihesus vocavit“ (,In virtute“ und „Probasti domine“) kombiniert, sondern erscheint zusammen mit den Antiphonen

36. Diese Handschrift enthält auch für die Vigil des Jacobusfestes ein Meßformular mit dem Introitus „Michi autem nimis“ (fol. 266v), so daß davon auszugehen ist, daß das Graduale „Nimis honorati“ auch dort gesungen wurde.

37. In F-Pn lat. 903 ist der Introitus „In omnem terram“ ohne Vers notiert. Da er aber in dieser Handschrift an anderen Stellen sowie auch in allen anderen herangezogenen Quellen immer in Verbindung mit dem Vers „Celi enarrant“ erscheint, wird auch hier von dieser Verbindung ausgegangen.

38. Codex Calixtinus, fol. 2v; Ripoll 99, fol. 2v.

39. Weitaus seltener ist dieser Introitus mit dem Graduale „Constitues eos“ verbunden, das im Codex Calixtinus für den zweiten Tag der Oktav vorgesehen ist (fol. 122v).

40. Die Communio „Ego vos elegi“ wurde in ganz Frankreich zu verschiedenen Apostel- und Märtyrerfesten gesungen. Sie erscheint in den unterschiedlichsten Kombinationen, so daß keine weiterführenden Aussagen möglich sind. 
„In omnem terram“, „Rorate caeli“, „Dominus secus“, „Lex domini“, „Mediatio cordis“ und „Dicit dominus“. Dort steht er bei den fünf erstgenannten im ersten und bei der letztgenannten im dritten Ton, nirgends aber wie in der Jacobusliturgie im siebten Ton. Daraus läßt sich schließen, daß die Kompilatoren aus einer Gegend stammten, in der er am Jacobusfest gesungen wurde, und ihn bewußt in dieses Meßformular einbezogen.

Auch an den drei dem Hauptfest des Jacobus vorangehenden Tagen, innerhalb der Oktav sowie am Oktavtag erklangen an verschiedenen Orten mehrere Gesänge, die sich in der Jacobusliturgie finden:

\section{Zentralfranzösische Notation:}

\begin{tabular}{|l|l|l|}
\hline F-Pn lat. 9437 (fol. 92r) & Com. „Tu es Petrus” & Ad vinc. S. Petri (1.8.) \\
\hline F-Pn lat. 12584 (fol. 190r) & Com. „Tu es Petrus” & Ad vinc. S. Petri (1.8.) \\
\hline F-Pn lat. 17328 (fol. 137r) & Off. „Angelus domini” & Maria Magdalena (22.7.) \\
\hline F-Psg 93 (fol. 164v) & Com. ,Tu es Petrus” & Ad vinc. S. Petri (1.8.) \\
\hline
\end{tabular}

\section{Aquitanische Notation:}

\begin{tabular}{|c|l|l|}
\hline B-Br II 3823 (fol. 105r) & Grad. „Ecce sacerdos” & Tr. Germani episc. (25.7) \\
\hline F-Pn lat. 776 (fol. 105r) & All. „Letabitur iustus” & Apollinaris episc. (23.7.) \\
\hline F-Pn lat. 780 (fol. 97r) & Grad. „Nimis honorati” & Ad vinc. S. Petri (1.8.) \\
(fol. 97r) & All. ,Letabitur iustus” & Felicis mart. (1.8.) \\
(fol. 96r) & Off. „Angelus domini” & Maria Magdalena (22.7.) \\
(fol. 97v) & Off. „Posuisti domine” & Felicis mart. (1.8.) \\
(fol. 97r) & Com. ,Tu es Petrus” & Ad vinc. S. Petri (1.8.) \\
\hline F-Pn lat.903(fol.105r/S.209) & All. „Letabitur iustus” & Apollinaris episc. (23.7.) \\
\hline
\end{tabular}

Teilweise wurden diese Gesänge, wie z.B. die Communio „Tu es Petrus“ zu Ad vincula S. Petri, an sehr vielen Orten mit einem bestimmten Fest in Verbindung gebracht. Teilweise sind sie auch nur in einzelnen Handschriften präsent, wobei sich eine Konzentration auf den aquitanischen Überlieferungskontext ablesen läßt.

Ein weiteres Kriterium für eine Melodieauswahl, auf das Johner hinwies, ist, daß ein ähnlicher Gedanke Festen oder Gesangsstücken zugrunde gelegen haben könnte ${ }^{41}$, d.h., daß es sich beispielsweise um Feste oder Gesänge für Heilige mit dem gleichen Status oder zum gleichen Anlaß - Feier der Nativitas, Passion usw. - handelte. Dieser Bezug wird auch in der Jacobusliturgie deutlich,

41. Johner: Wort und Ton, S.462. 
indem mehrere der Melodievorlagen zu Apostel- und Märtyrerfesten wie etwa zu Laurencii martyris, Stephani martyris, Valentini martyris, Andreae apostoli, Bartholomei apostoli, Thomae apostoli usw. gesungen wurden oder in den Märtyrercommune enthalten sind. Ein großer Teil der Melodievorlagen findet sich in Handschriften beider Traditionen. Eindeutig in den aquitanischen Bereich weist jedoch das Offertorium „Iustorum anime“, das innerhalb der hier herangezogenen Handschriften in keiner zentralfranzösischen, dafür aber in mehreren aquitanischen Quellen enthalten ist: in E-Mah Aemil. 18 (fol. 297v/fol. 298r) zu Nativitas plur. mart. ${ }^{42}$, in F-pn lat. 780 (fol. 98r) zu Justi et Pastoris mart. sowie in F-Pn lat. 776 (fol. 118v/119r), F-Pn lat. 903 (fol. 112r/S. 223) und GB-Lbl Harley 4951 (fol. 289) zu Omnium sanctorum. In letzteren drei Handschriften erscheint dieser Gesang in Verbindung mit dem Introitus „Gaudeamus omnes“. Dieser ist wiederum in der einleitenden Epistel im Codex Calixtinus und der Handschrift Ripoll 99 als einer der Gesänge genannt, die ursprünglich in Santiago de Compostela zum Jacobusfest gesungen wurden ${ }^{43}$.

Bezüglich der Cluniazenser Handschriften ergab sich im Hinblick auf die Auswahl der Melodievorlagen kein Hinweis auf eine Verbindung. Mit den oben erwähnten Auswahlkriterien korrespondierten nur die Gesänge, die zum Standardrepertoire des Jaocbusfestes (Graduale „Nimis honorati“) und von Ad vincula S. Petri (Communio „Tu es Petrus“) gehören.

Sowohl die Melodievorlagen, die am Jacobusfest selbst oder in seinem zeitlichen Umkreis gesungen wurden, als auch die, deren Zuordnung zu einem Fest eine Parallele zur Jacobusliturgie erkennen läßt, lassen annehmen, daß die Kompilation aus dem aquitanischen Raum stammt und in direktem Zusammenhang mit Santiago de Compostela entstand. Somit liegt der Schluß nahe, daß es sich beim Codex Calixtinus nicht um die Urfassung des „Liber Sancti Iacobi“, sondern um eine in Zentralfrankreich angefertigte Redaktion handelt. Diese Annahmen korrespondieren mit den eingangs erwähnten und in der Forschung oft nicht ganz ernst genommenen Thesen Hohlers.

Die Abweichungen zwischen den Melodiefassungen im Codex Calixtinus und in Ripoll 99 beziehen sich teils auf Einzeltöne, teils auf mehrere aufeinanderfolgende Töne. Es stellt sich die Frage, ob sich aus ihrer Art und ihrem Auftreten Schlüsse hinsichtlich des Verhältnisses zwischen beiden Quellen ziehen lassen.

Varianten bei Liqueszenzen und Zierneumen, Strophici und Tonrepetitionen sowie im Bereich der Halbtöne mi-fa kommen meist in der gleichen Art und Weise vor und können deshalb als generelle Unterschiede zwischen beiden Quellen betrachtet werden. So ist der folgende kurze Ausschnitt aus dem Alleluia „Sanctissime apostole ${ }^{\text {“44 }}$ symptomatisch für das Auftreten von Tonrepetitionen in den beiden Handschriften: Im Codex Calixtinus wird allein innerhalb eines Melismas zweimal das d' bei der Abschnittsgliederung wiederholt, während es in Ripoll 99 jeweils nur einmal erscheint.

42. In E-Mah $51 \mathrm{zu}$ „Simonis et Jude apostolorum“.

43. Codex Calixtinus, fol. $2 v$. Aufgrund dieser liturgischen Zuordnung kann davon ausgegangen werden, daß „Iustorum anime“ und nicht das auf der gleichen Melodie basierende „Stetit angelus“, für das sich keine vergleichbaren Kriterien feststellen lassen, als Vorlage gedient hat.

44. Codex Calixtinus, fol. 119r; Ripoll 99, fol. 32r. 


\section{Beispiel 1:}

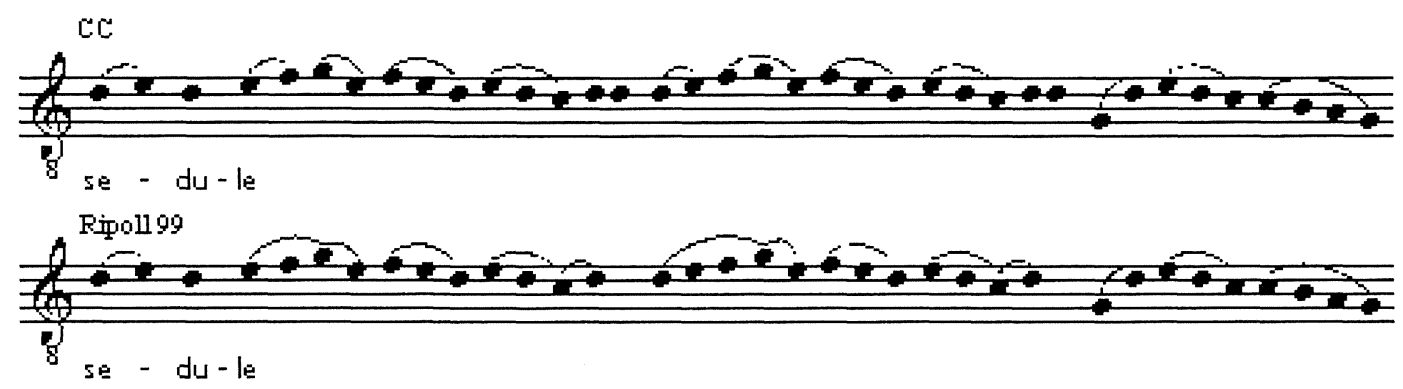

Dagegen weist Ripoll 99 insgesamt wesentlich mehr Liqueszenzen auf als der Codex Calixtinus $^{45}$. Bei Abweichungen im Bereich der Halbtöne mi-fa erscheint in Ripoll 99 jeweils der höhere Ton.

Alle anderen Abweichungen lassen sich nicht klassifizieren. So zeigt das folgende Beispiel aus

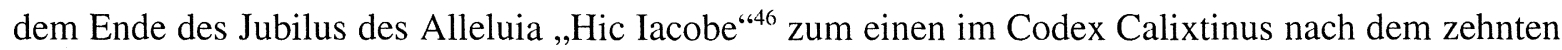
Ton ein zusätzliches e, das die in Ripoll 99 notierte Terz d-f ausfüllt, und zum anderen in Ripoll 99 nach dem dreizehnten Ton ein zusätzliches d':

\section{Beispiel 2:}

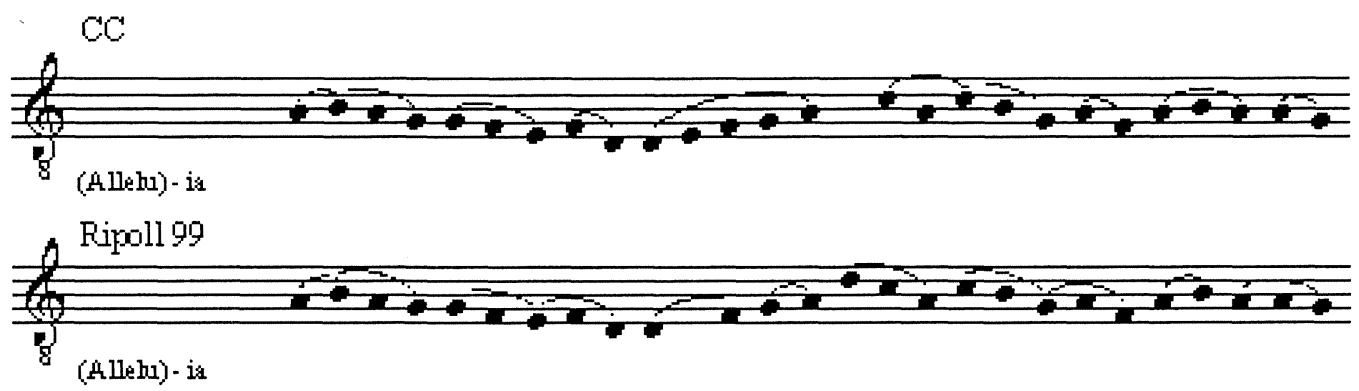

Während die ersten beiden Beispiele aus adaptierten Gesängen stammen, ist das folgende dem neu komponierten Vers zum Offertorium „Ascendens Ihesus“ entnommen ${ }^{47}$. Es weist gleich mehrere

45. Eine Rolle spielen hier nur die Liqueszenzen, die zu zusätzlichen Tönen führen und nicht nur eine modifizierte Notationsweise darstellen.

46. Codex Calixtinus, fol. 119r; Ripoll 99, fol. 32r.

47. Codex Calixtinus, fol. 121v; Ripoll 99, fol. 33r. 
Abweichungen auf, die sich nicht nur auf Einzeltöne, sondern auch auf ganze Tongruppen und Textsilben beziehen und teils genereller Art sind, teils nicht:

\section{Beispiel 3}
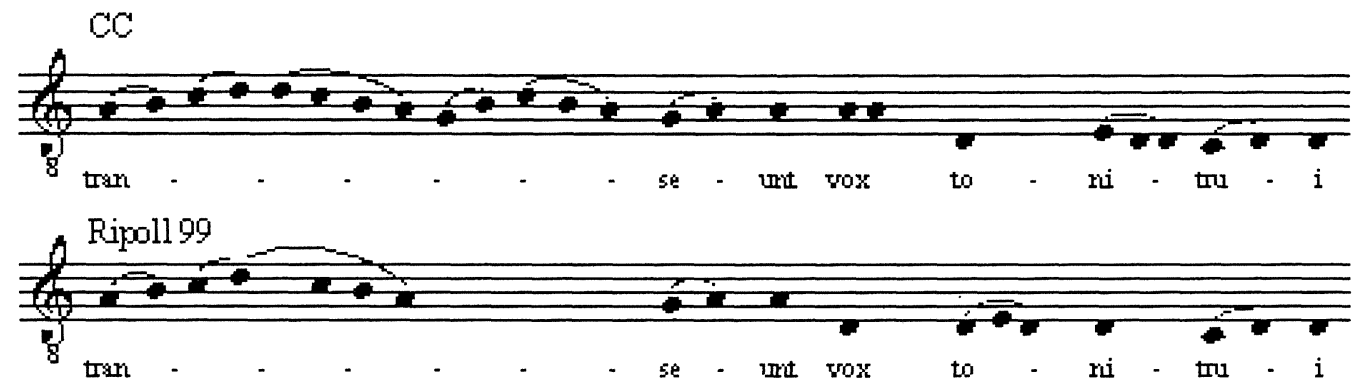

Wenn Arnaldus tatsächlich den heutigen Codex Calixtinus vor sich hatte, müßte ihm unterstellt werden, daß er mit seiner Vorlage sehr frei umging. Bei den Adaptionen könnte er die Melodien gemäß seiner Erinnerung an ihm vertraute Melodiefassungen notiert haben. Bei den Neukompositionen - die keineswegs weniger Abweichungen enthalten als die Adaptionen - würde diese Möglichkeit jedoch wegfallen. Der Schreiber könnte sich entweder an entsprechenden Elementen in ihm bekannten Gesängen orientiert oder individuelle Entscheidungen getroffen, also nicht notengetreu abgeschrieben haben. Allerdings fällt die Konsequenz auf, mit der die generellen Abweichungen sowohl in adaptierten als auch in neu komponierten Gesängen auftreten. Diese Beobachtung ist nicht in Übereinklang zu bringen mit der relativ flüchtig wirkenden Notation von Ripoll 99, die oft unregelmäßige Abstände zwischen den Neumen und zahlreiche Silbenzuordnungsstriche aufweist und eher auf einen Schreiber hindeutet, der unter Zeitdruck stand, als auf einen, der in aller Ruhe den Notentext redigieren konnte ${ }^{48}$. So liegt die Vermutung nahe, daß Ripoll 99 nicht nur keine genaue Kopie des Codex Calixtinus ist, sondern auf einer anderen Vorlage basiert.

Ein weiterer Schritt zur Klärung der angesprochenen Fragen ist der Versuch, die Abweichungen zwischen den beiden Melodiefassungen, den in beiden Quellen enthaltenen Melodiebestand sowie auch die Gesänge der Vigilmesse in den zentralfranzösischen oder in den aquitanischen Überlieferungskontext einzuordnen. Wenn nämlich die angesichts der Auswahl der Melodievorlagen formulierte These zur Entstehung der Liturgie und des Codex Calixtinus zuträfe, müßten das beiden Handschriften gemeinsame melodische Material, wie auch die Melodiefassungen in Ripoll 99 in den aquitanischen und die Varianten des Codex Calixtinus in den zentralfranzösichen Raum weisen. Da

48. Mit dieser Annahme korrespondiert Hämels Hinweis (Überlieferung, S. 28), daß Arnaldus wohl wegen Zeitmangels aus dem „Liber Sancti Iacobi“ nur Exzerpte anfertigte. 
die Vigilmesse nur im Codex Calixtinus überliefert ist, stellt sich die Frage, ob sie für diese Handschrift neu geschaffen wurde oder ob sie bereits in früheren Redaktionen des „Liber Sancti Iacobi“" enthalten war. Im ersten Fall müßte sie der zentralfranzösischen, im zweiten der aquitanischen Tradition zuzuordnen sein.

Der Vergleich der Abweichungen in den auf ältere Melodien zurückgehenden Gesängen mit den am besten lesbaren und vollständigsten der bereits verwendeten Quellen ergab folgendes Bild: Innerhalb der herangezogenen Handschriften ließ sich keine finden, die eindeutig das Umfeld der Kompilatoren oder auch der Schreiber von Codex Calixtinus und Ripoll 99 repräsentiert. Dennoch ließen sich Entsprechungen feststellen, die eine Einordnung in die beiden Überlieferungskontexte zulassen:

Der folgende Ausschnitt aus dem Introitus ,Ihesus vocavit ${ }^{\text {“49 }}$ zeigt einige - vor allem am Ende deutliche - Unterschiede zwischen den beiden Melodiefassungen. Für das Melisma über ,,(Bo)-ạ(nerges)“ im Codex Calixtinus findet sich nur in der zentralfranzösischen Handschrift F-Pn lat. 9435 (fol. 237v) im Introitus „In virtute“ bei ,ve-(hementer)“ eine tongetreue Entsprechung. Die aquitanischen Überlieferungen B-Br II 3823 (fol. 108v), E-Mah 18 (fol. 276r), F-Pn lat. 776 (fol. 109v), F-Pn lat. 780 (fol. 99r), F-Pn lat. 903 (fol. 109/S. 217), GB-Lbl Harley 4951 (fol. 280r/v) des auf der gleichen Melodie basierenden Introitus „Probasti me“ zeigen bei „(exa)-mi-(nasti)“ ein einheitliches Bild, das von der Fassung in Ripoll 99 nur durch das Fehlen des vierten Tones h in dem

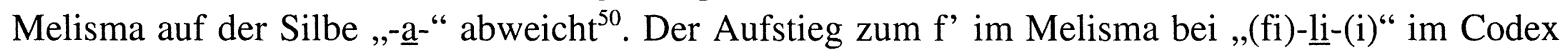
Calixtinus entspricht ,,(desi)-de-(rium)“ in ,In virtute“ nur in der zentralfranzösischen Handschrift FPn lat. 17328 (fol. 158v) ) $^{51}$. Mit Ripoll 99 stimmt bei ,fi-(lii)“ nur „Probasti domine“ bei „non“ in der aquitanischen Quelle E-Sa 2637 (fol. 210v/211r) überein:

49. Codex Calixtinus, fol. 118r; Ripoll 99, fol. 31v.

50. In Ripoll 99 ist hier nicht zweifelsfrei erkennbar, ob es sich beim vorletzten Ton des Melismas auf „-a-“ um eine Note oder wie in den aquitanischen Quellen um ein Quilisma handelt.

51. Eine ähnliche Wendung findet sich in „Iudicant sancti“, das Wagner als Vorlage für den Schluß in Erwägung zieht. Da es keine weiteren Gemeinsamkeiten zwischen „Ihesus vocavit“ und „Iudicant sancti“ gibt, ist eine Beziehung zu diesem Gesang unwahrscheinlich. 


\section{Beispiel 4:}

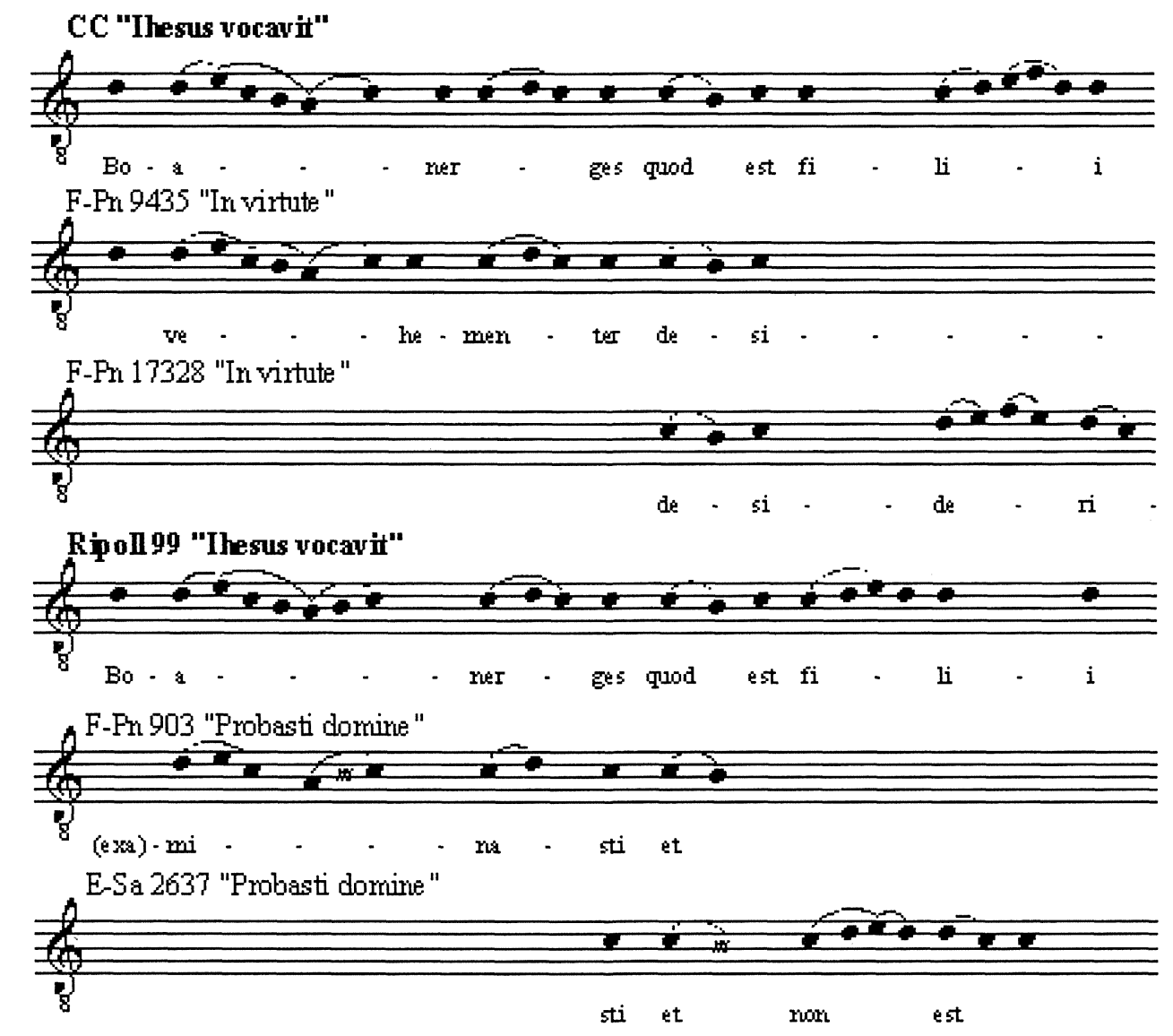

Auf der einen Seite erlauben die beschriebenen Korrespondenzen zu den verschiedenen Überlieferungen eine dem Notationsbefund entsprechende Zuordnung des Codex Calixtinus zum zentralfranzösichen und von Ripoll 99 zum aquitanischen Kontext. Auf der anderen Seite berücksichtigt nur die Version in Ripoll 99 den natürlichen Sprachfall des Wortes „filii“. Aufgrund dieses Sachverhaltes hält Wulf Arlt die Fassung in Ripoll 99 für die ,adäquatere Formulierung“ und zieht für den Codex Calixtinus eine „Entstellung“ der Kopie in Betracht. Er erinnert an Wagners Verweis auf „die Möglichkeit einer Orientierung des Arnaldus an ihm vertrauten Formulierungen“52. Hier gilt es zu bedenken, daß der Urheber der Melodiefassung im Codex Calixtinus bei ,filii““ möglicherweise bewußt eine Änderung gegenüber seiner Vorlage vornahm, um das Ende der Antiphon durch den Aufstieg zum f' in Beziehung zur kleinen Doxologie am Schluß des anschließenden Psalmverses „Celi enarrant“ zu setzen:

52. Arlt: „Rezeption“, S. 415; vgl. Wagner: Die Gesänge, S. 9, 146. 


\section{Beispiel 5:}

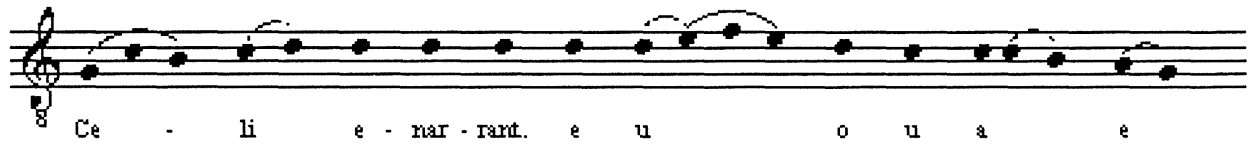

Eine so eindeutige Zuordnung wie in Beispiel 4 ist jedoch längst nicht bei allen insgesamt festgestellten 66 Abweichungen möglich: 17 finden sich in Neukompositionen oder bei gegenüber den Melodievorlagen hinzugefügten Tönen und fallen für diese Auswertung nicht ins Gewicht. Die übrigen 49 verteilen sich wie folgt:

\begin{tabular}{|l|l|l|}
\hline & Codex Calixtinus & Ripoll 99 \\
\hline zentralfranzösische Quellen & 17 & 4 \\
\hline aquitanische Quellen & 4 & 17 \\
\hline verschiedene Quellen beiderlei Provenienz & 18 & 7 \\
\hline keine Korrespondenzen & 10 & 21 \\
\hline
\end{tabular}

Ein Überblick über die Zuordnung jeder einzelnen Abweichung ergab, daß der Codex Calixtinus am häufigsten den Handschriften F-Pn nouv. acq. lat. 1235 aus Nevers und F-Pn lat. 17328 aus Paris entsprach. Der deutliche Hinweis auf Nevers korrespondiert mit dem Notationsbefund. Für Ripoll 99 ergaben sich die meisten Korrespondenzen gegenüber der Handschrift F-Pn lat. 776 aus Albi, gefolgt von F-Pn lat. 903 aus Saint-Yrieix.

Überraschenderweise läßt sich der gemeinsame melodische Bestand von Codex Calixtinus und Ripoll 99 nicht wie aufgrund der Melodieauswahl angenommen, in den aquitanischen Kontext einordnen, sondern korrespondiert sowohl mit aquitanischen als auch mit zentralfranzösischen Handschriften. Die meisten Entsprechungen ergaben sich gegenüber F-Pn nouv. acq. lat. 1235 aus Nevers, gefolgt von F-Pn lat. 903 aus Saint-Yrieix.

Auch die Melodiefassungen der Vigilmesse lassen Einflüsse beider Traditionen erkennen. Es fanden sich ungefähr gleich viele Bezüge zu F-Pn lat. 10511 aus Auxerre und F-Pn lat. 17328 aus Paris, E-SA 2637 aus Salamanca (?), F-Pn nouv. acq. lat. 1235 aus Nevers, E-Mah Aemil. 18 aus S. Millán de la Cogolla, F-Pn lat. 903 aus Saint-Yrieix und F-Pn lat. 776 aus Albi. Aufgrund der dem gemeinsamen Bestand entsprechenden Einordnung ihrer Melodiefassungen in die beiden Überlieferungskontexte läßt sich kein Hinweis darauf finden, daß die Vigilmesse in den früheren Redaktionen des „Liber Sancti Iacobi“ sowie auch in der für Ripoll 99 verwendeten Vorlage nicht 
enthalten gewesen wäre. Möglicherweise verzichtete Arnaldus - wie auch bei anderen Abschnitten des „Liber Sancti Iacobi“ - aus Zeitmangel auf eine Abschrift ${ }^{53}$.

Bei einem großen Teil der Abweichungen zwischen Codex Calixtinus und Ripoll 99 läßt sich eine der Notation entsprechende Zuordnung zu den Überlieferungskontexten ablesen. Der andere Teil sowie auch der gemeinsame melodische Bestand und die Vigilmesse sind teils gar nicht einzuordnen, teils enthalten sie Elemente beider Traditionen. Die Stellen, die sich in keinen Kontext einordnen lassen, beruhen - sofern sie sich nicht in neukomponierten Abschnitten befinden - entweder darauf, daß die Melodiefassungen auf Handschriften zurückgehen, die hier nicht untersucht wurden, oder daß Momente individuellen Gestaltens von Seiten der Kompilatoren oder Schreiber vorliegen. Die Varianten, die auf die jeweils andere Tradition zurückzuführen sind, können als ein Hinweis darauf interpretiert werden, daß sie nicht erst auf die beiden Schreiber, die ja vermutlich nur mit ihrer eigenen Umgebung vertraut waren, zurückzuführen sind, sondern daß in beiden Fällen auf Material, das bereits in unterschiedlichen Kontexten redigiert worden war, zurückgegriffen wurde.

Zusammenfassend spricht vieles dafür, daß die Handschrift Ripoll 99 keine Abschrift des Codex Calixtinus ist und auch nicht auf der selben Vorlage basiert wie dieser. Es ist anzunehmen, daß beide Handschriften auf verschiedene, nicht erhaltene Überlieferungen des „Liber Sancti Iacobi“ zurückgehen. Daraus folgt auch, daß der Codex Calixtinus nicht zwangsläufig vor der Handschrift Ripoll 99 entstanden sein muß, wie es bis jetzt vielfach angenommen wurde ${ }^{54}$.

Der Umstand, daß die Auswahl der Melodievorlagen auf den aquitanischen Bereich hinweist, ist als Indiz für eine ursprünglich spanische oder südfranzösische Provenienz des „Liber Sancti Iacobi“ zu deuten. Doch bereits der dem Codex Calixtinus und Ripoll 99 gemeinsame melodische Bestand sowie die Melodien der Vigilmesse lassen außer dem aquitanischen einen zentralfranzösischen Einfluß erkennen. So liegt hier ein Hinweis darauf vor, daß es bereits vor den beiden hier behandelten Quellen Redaktionen gab, die in Zentralfrankreich oder von einem von dort stammenden Redaktor vorgenommen wurden. Als regionale Eingrenzung für die Urfassung ließ sich aufgrund der Auswahl der Melodievorlagen eine Tendenz nach Narbonne, Albi und Saint-Yrieix ausmachen, wobei die Kompilatoren Kenntnis von der liturgischen Tradition Santiago de Compostelas hatten. Die Redaktion des Codex Calixtinus könnte in Nevers, Auxerre oder Paris vorgenommen worden sein, wobei die Neumenschrift nach Nevers weist. Dieses Bild korrespondiert in mehreren Punkten mit den mehrfach erwähnten Thesen Hohlers.

53. Die Suche nach weiteren Gründen für das Fehlen der Vigilmesse in Ripoll 99 würde weitere Untersuchungen verlangen und den Rahmen dieses Textes sprengen. musica, S. 88.

54. Vgl. z.B. Hämel: Überlieferung, S. 14; Herbers: Der Jakobuskult, S. 33; Villaverde: La liturgia, S. 35; López-Calo: La 
VERENA FÖRSTER BINZ

\title{
Bibliographie
}

\author{
Handschriften: ${ }^{55}$
}

B-Br II 3823: Bruxelles, Bibliothèque Royal Albert Ier, II 3823

E-Bac, Ripoll 99: Barcelona, Arxiu de la Corona d'Aragón, Ripoll 99

E-Mah Aemil. 18: Madrid, Archivo Historico Nacional, Aemil. 18

E-Mah Aemil. 51: Madrid, Archivo Historico Nacional, Aemil. 51

E-SA 2637: Salamanca, Biblioteca Universitaria, ms. 2637

E-SCc s.s.: Santiago de Compostela, Archivo del Cabido de la Catedral, Codex Calixtinus, sin número F-A 181: Avignon, Bibliothèque municipale, ms. 181

F-AN 97 (89): Angers, Bibliothèque municipale, ms. 97 (89)

F-Pn lat. 776: Paris, Bibliothèque nationale, fond lat. 776

F-Pn lat. 780: Paris, Bibliothèque nationale, fond lat. 780

F-Pn lat. 903: Paris, Bibliothèque nationale, fond lat. 903

F-Pn lat. 1087: Paris, Bibliothèque nationale, fond lat. 1087

F-Pn lat. 9435: Paris, Bibliothèque nationale, fond lat. 9435

F-Pn lat. 10511: Paris, Bibliothèque nationale, fond lat. 10511

F-Pn lat. 12584: Paris, Bibliothèque nationale, fond lat. 12584

F-Pn lat. 17328: Paris, Bibliothèque nationale, fond lat. 17328

F-Pn nouv. acq. lat. 1235: Paris, Bibliothèque nationale, nouv. acq. fond lat. 1235

GB-Lbl Harley 4951: London, British Library, ms. Harley 4951

\section{Editionen:}

Helmer, Paul (hrsg.): The Mass of St. James, Ottawa 1988 (Wissenschaftliche Abhandlungen 49)

Herbers, Klaus und Noia, Manuel Santos (hrsg.): Liber Sancti Iacobi Codex Calixtinus, Santiago de Compostela 1997/98

López-Calo, José (hrsg.): La Musica en la Catedral de Santiago, Vol. V, La Edad Media, La Coruna 1994

Lozano, Millán Bravo und Kaydeda, José Maria (hrsg.): Jacobus. Codex Calixtinus de la Catedral de Santiago de Compostela (Faksimile), Santiago de Compostela 1993

Wagner, Peter (hrsg.): Die Gesänge der Jakobusliturgie zu Santiago de Compostela aus dem sog. Codex Calixtinus, Freiburg/Schweiz 1931 (Collectanea Friburgensia 29)

Whitehill, Walter Muir und Prado, Germán (hrsg.): Liber Sancti Iacobi. Codex Calixtinus, Bd. I-III, Santiago de Compostela 1944

55. Den Verantwortlichen des Mikrofilmarchivs des Musikwissenschaftlichen Instituts der Universität Basel danke ich herzlich für die Unterstützung. 


\section{Literatur:}

Anglès, Higini: La música a Catalunya fins al segle XIII, Barcelona 1935

Arlt, Wulf: „Rezeption, Adaption und Redaktion in den Gesängen des Jacobusoffiziums im Kontext musikalisch-liturgischer Festgestaltung des 12. Jahrhunderts“, in: El Códice Calxtino y la música de su tiempo. Actas del simposio organizado por la Fundación Pedro Barrié de la Maza en La Coruña y Santiago de Compostela, 20-23 de septiembre de 1999, La Coruña 2001, S. 387-442

Bédier, José: Les légendes épiques. Recherches sur la formation des chansons de geste, Bd. III, Paris 1912

Diaz y Diaz, Manuel C.: El codice calixtino de la catedral de Santiago. Estudio codicológico y de contenido, Santiago de Compostela 1988 (Centro de estudios jacobeos. Monografias de compostellanum 2)

Fuller, Sarah: „Perspectives on Musical Notation in the Codex Calixtinus“, in: El Códice Calxtino y la música de su tiempo. Actas del simposio organizado por la Fundación Pedro Barrié de la Maza en La Coruña y Santiago de Compostela, 20-23 de septiembre de 1999, La Coruña 2001, S. 183-234

Hämel, Adalbert: Überlieferung und Bedeutung des Liber Sancti Jacobi und des Pseudo-Turpin, München 1950 (Sitzungsberichte der Bayerischen Akademie der Wissenschaften, Philosophisch-historische Klasse 1950/2)

Herbers, Klaus: Der Jakobuskult des 12. Jahrhunderts und der „Liber Sancti Jacobi“. Studien über das Verhältnis zwischen Religion und Gesellschaft im hohen Mittelalter, Wiesbaden 1984 (Historische Forschungen VII)

Hiley, David und Emerson, John A.: Art. „Sources, 2. Manuscripts of Western Plainchant“, in: New Grove of Music and Musicians (2001), S. 824-839

Hohler, Christopher: „A Note on Jacobus”, in: Journal of the Warburg and Courtauld Institutes 35 (1972), S. 31-80

Huglo, Michel: „Les pièces notées du Codex Calixtinus“, in: The Codex Calixtinus and the Shrine of St. James, Tübingen 1992, S. 105-124 (Jakobus-Studien 3)

Huglo, Michel: Rezension zu André Moisan: Le livre de Saint Jacques, in: Scriptorium 47, 1993/1, S. 259-260

Huglo, Michel: „The Origin of the Monodic Chants in the Codex Calixtinus“, in: Essays on Medieval Music, in Honor of David G. Hughes, Cambridge (Massachusetts) 1994, S. 195-205

Huglo, Michel und Ferreira, Manuel Pedro: Art. „Cluniac monks, 2. Cluniac manuscripts of mass chants“, in: The New Grove Dictionary of Music and Musicians (2001), Bd. VI, S. 63-65.

Johner, Domenicus: Wort und Ton im Choral. Ein Beitrag zur Ästhetik des gregorianischen Gesanges, Leipzig 1940

Lara, F. Javier: „Estructuras modales en el Códice Calixtino“, in: El Códice Calxtino y la música de su tiempo. Actas del simposio organizado por la Fundación Pedro Barrié de la Maza en La Coruña y Santiago de Compostela, 20-23 de septiembre de 1999, La Coruña 2001, S. 273-307 
López-Calo: „Claves“, in: El Códice Calxtino y la música de su tiempo. Actas del simposio organizado por la Fundación Pedro Barrié de la Maza en La Coruña y Santiago de Compostela, 20-23 de septiembre de 1999, La Coruña 2001, S. 235-271

Lütolf, Max: Die mehrstimmigen Ordinarium-Sätze vom ausgehenden 11. bis zur Wende des 13. zum 14. Jahrhundert, Bd. I-II, Bern 1970

Moisan, André: Le Livre de Saint Jacques ou Codex Calixtinus de Compostelle. Etude critique et littéraire, Genève 1992

Schlager, Karlheinz: Alleluia-Melodien I, bis 1100, Kassel u.a. 1968 (Monumenta Monodica Medii Aevi Bd. VII)

Schlager, Karlheinz: Alleluia-Melodien II, ab 1100, Kassel u.a. 1987 (Monumenta Monodica Medii Aevi Bd. VIII)

Schlager: Art. „Alleluia“, in: Die Musik in Geschichte und Gegenwart², Sachteil, Bd. I (1994), Sp. 445-459

Villaverde, Elisardo Temperàn: La Liturgia Propia de Santiago en el Códice Calixtino, Santiago de Compostela 1997

Wagner, Peter: Einführung in die Gregorianischen Melodien, Bd. I-III, Leipzig 1911-1921

Wagner, Peter: „Wie man im Mittelalter eine neue Choralmesse komponierte“, in: Gregoriusblatt 51/9-11 (1927), S. 129-134, 145-150, 161-169 